\title{
A Novel and Efficient Synthesis of [1,2,3]-Triazolyl Substituted Benzo[c]Coumarins and Evaluation of their Antimicrobial Activity
}

\author{
Neha N. Gohil, Kaushik N. Kundaliya, D. I. Brahmbhatt* \\ Department of Chemistry, Sardar Patel University, Vallabh Vidyanagar-388120, Gujarat, India \\ *E-mail: drdib317@gmail.com
}

Keywords: [1,2,3]-triazole, benzo[c]coumarin, antimicrobial activity, broth dilution method.

\begin{abstract}
A series of novel [1,2,3]-triazolyl substituted benzo[c]coumarins have been synthesized by reacting various 3-coumarinoyl methyl pyridinium bromide salts with 1-(5-methyl-1-phenyl-1H1,2,3-triazol-4-yl)ethanones in the presence of sodium acetate in refluxing acetic acid. The structures of the synthesized compounds have been elucidated by IR, ${ }^{1} \mathrm{H}-\mathrm{NMR},{ }^{13} \mathrm{C}-\mathrm{NMR}$ and Mass spectral data. All the synthesized compounds have been screened for their in vitro anti-bacterial and anti-fungal activities. Some of the compounds have been found to be active against some bacterial and fungal pathogens compared to standard drugs.
\end{abstract}

\section{Introduction}

Benzo $[c]$ coumarins form an important class of coumarin derivatives and are isolated from plant and animal sources and are mainly metabolic products of microorganisms [1]-[3]. In literature many reports are there for isolation of natural products having benzo[c]coumarin skeleton such as urolitin B [4,5], alternariol [6], autumnariol, autumnariniol [7], altenuisol [8], arnottin [9], defucogilvocarcin [10] etc. Certain benzo[c]coumarins are reported to be used as an intermediate for the synthesis of variety of pharmaceutically interesting compounds including progesterone, androgen, glucocorticoid modulators [11]-[13], endothelial cell proliferation inhibitors [14] and anti-dyslipidemic agents [15]. Many benzo[c]coumarin derivatives are reported to possess various antibiotic [16], anticancer [17], antiviral [18], anti-mutagenic [19], anti-proliferative [20], antiallergic [21], cytotoxic [22], AChE inhibitory activity [23], antioxidant and free radical scavenging activity [24] etc.

A literature survey for the synthesis of benzo[c]coumarins revealed that in majority of the reports on the synthesis of benzo[c]coumarins, the compounds have been synthesized by the lactonization of appropriately substituted biphenyl derivatives [25]-[27]. In the present work we have synthesized some new benzo[c]coumarin derivatives utilizing a novel and distinct approach.

Triazoles form an important class in heterocyclic chemistry. Many [1,2,3]-triazole derivatives are known for their varied biological activity such as anti-HIV [28], antimicrobial [29], antihistaminic [30], anti-proliferative [31], antiviral and cytotoxic properties [32], antiinflammatory agents [33], $\beta$-lactamase inhibitors [34], agonists of muscarine receptors [35], antagonists of GABA receptors [36] etc. In addition to above biological activities certain [1,2,3]triazoles are also known for their fungicidal and plant growth regulator properties and are used as agrochemicals. Certain triazole derivatives are also reported to be used in industry as dyes, corrosion inhibitors and photo stabilizers [37].

Thus considering the importance of benzo[c]coumarins and triazoles, it was thought worthwhile to synthesize a new class compounds which are hybrid of benzo[c]coumarin and $[1,2,3]$-triazole. Keeping this objective in mind in the present work we have synthesized some $[1,2,3]$-triazolyl substituted benzo[c]coumarins using a new synthetic approach.

\section{Experimental}

All the melting points are uncorrected. All reactions were performed with commercially available reagents and they were used without further purification. Organic solvents were purified by standard methods and stored over molecular sieves. All the IR spectra ( $\mathrm{KBr}$ disc) were recorded 
on Shimadzu FT-IR 8400-S spectrometer. ${ }^{1} \mathrm{H}$ NMR and ${ }^{13} \mathrm{C}$ NMR spectra were recorded on Bruker Advance 400 spectrometer operating at $400 \mathrm{MHz}$ for ${ }^{1} \mathrm{H}$ NMR and $100 \mathrm{MHz}$ for ${ }^{13} \mathrm{C} \mathrm{NMR}$. The chemical shift $(\delta)$ is reported in ppm using chloroform-d as a solvent and calibrated standard solvent signal. Mass spectra were recorded on Shimadzu QP 2010 spectrometer. Elemental analysis was carried out on Perkin- Elmer 2400 C-H-N-S-O Analyzer Series-II. Column chromatography was performed with silica gel 60-120 mesh (Merck, Mumbai, India.). All the compounds were routinely checked for completion of the reaction on silica gel 60 F254 TLC plates and their spots were visualized by exposure to a UV lamp, iodine vapour or $\mathrm{KMnO} 4$ reagents. Compounds 3-coumarinoyl methyl pyridinium bromide salts (1a-c) [38]-[40] and 1-(5-methyl-1-aryl-1H-1,2,3triazol-4-yl)ethanones (2a-d) [41] were prepared according to literature procedure.

\subsection{General procedure for the synthesis of 7-hydroxy-9-(5-methyl-1-aryl-1H-1,2,3-triazol-4- yl) benzo $[c]$ coumarins (3a-l)}

A solution of an appropriate 3-coumarinoyl methyl pyridinium bromide salt (1a-c) $(0.004$ $\mathrm{mol})$ in glacial acetic acid $(40 \mathrm{~mL})$ was added in a round bottom flask. To this, sodium acetate (0.012 mol) and appropriate 1-(5-methyl-1-aryl-1H-1,2,3-triazol-4-yl)ethanone (0.004 mol) (2a-d) was added with stirring at room temperature during 10 minutes. The reaction mixture was further stirred for 20 minutes at room temperature and then refluxed for 8 hours. It was then allowed to cool to room temperature and poured into ice-cold water and extracted with chloroform $(3 \mathrm{x}$ $30 \mathrm{~mL})$. The combined chloroform extract was washed with water $(2 \times 20 \mathrm{~mL})$ and then dried over anhydrous sodium sulphate. The removal of chloroform under reduced pressure gave a solid product, which were subjected to column chromatography using chloroform-petroleum ether (60-80) (8:2) as an eluent to obtain the pure product (3a-I) (scheme-1).

\subsection{Analytical and Spectral Characterization}

The structure of all the twelve synthesized (3a-l) compounds were confirmed by their ${ }^{1} \mathrm{H}-\mathrm{NMR},{ }^{13} \mathrm{C}$ - NMR, IR, elemental analysis and representative mass spectral data given below.

7-Hydroxy-9-[5'-methyl-1'-phenyl-1 H-1,2,3-triazole-4'-yl]benzo[c]coumarin (3a): White solid; yield $=80 \%$; mp 227-229 ${ }^{\circ} \mathrm{C}$; Anal. Calcd. For $\mathrm{C}_{22} \mathrm{H}_{15} \mathrm{~N}_{3} \mathrm{O}_{3}: \mathrm{C}, 71.54 ; \mathrm{H}, 4.09 ; \mathrm{N}, 11.38 \%$. Found: C, 71.58; H, 4.14; N, 11.42\%. IR $\left(\mathrm{KBr}, v_{\max }, \mathrm{cm}^{-1}\right) ; 1680(\mathrm{C}=\mathrm{O}$ stretching of $\delta$-lactone of coumarin), 1628 (aromatic $\mathrm{C}=\mathrm{C}$ stretching), 3068 (aromatic $\mathrm{C}-\mathrm{H}$ stretching), 2923 (aliphatic $\mathrm{C}-\mathrm{H}$ stretching), 3443 (O-H stretching). ${ }^{1} \mathrm{H}$ NMR $\left(400 \mathrm{MHz}, \mathrm{CDCl}_{3}, \delta\right): 2.62\left(3 \mathrm{H}\right.$, singlet, $\mathrm{CH}_{3}$ of triazole ring), 7.38-8.28 $(11 \mathrm{H}$, multiplet, aromatic protons $), 11.48\left(1 \mathrm{H}\right.$, singlet, -OH proton). ${ }^{13} \mathrm{C}$ NMR $\left(100 \mathrm{MHz}, \mathrm{CDCl}_{3}, \delta\right): 10.75\left(\mathrm{CH}_{3}\right), 105.05(\mathrm{C}) 111.10(\mathrm{CH}), 113.63(\mathrm{CH}), 117.75(\mathrm{CH})$, $118.30(\mathrm{C}), \quad 123.76(\mathrm{CH}), \quad 125.29(\mathrm{CH}), 125.47(\mathrm{CH}), 129.73(\mathrm{CH}), 129.96(\mathrm{CH}), 130.87(\mathrm{CH})$, 131.62(C), 135.71(C), 135.90(C), 140.25(C), 143.01(C), 150.75(C), 162.62(C), 165.31(CO of coumarin).

7-Hydroxy-9-[5'-methyl-1'-(p-tolyl)-1H-1,2,3-triazole-4'-yl]benzo[c]coumarin (3b): White solid; yield $=82 \%$; mp 206-208 ${ }^{\circ} \mathrm{C}$; Anal. Calcd. For $\mathrm{C}_{23} \mathrm{H}_{17} \mathrm{~N}_{3} \mathrm{O}_{3}$ : C, 72.05; H, 4.47; N, 10.96\%. Found: C, $72.01 ; \mathrm{H}, 4.42 ; \mathrm{N}, 10.92 \%$. IR $\left(\mathrm{KBr}, v_{\max }, \mathrm{cm}^{-1}\right) ; 1682(\mathrm{C}=\mathrm{O}$ stretching of $\delta$-lactone of coumarin), 1620 (aromatic $\mathrm{C}=\mathrm{C}$ stretching), 3070 (aromatic $\mathrm{C}-\mathrm{H}$ stretching), 2932 (aliphatic $\mathrm{C}-\mathrm{H}$ stretching), 3418 (O-H stretching). ${ }^{1} \mathrm{H}$ NMR $\left(400 \mathrm{MHz}, \mathrm{CDCl}_{3}, \delta\right): 2.50$ (3H, singlet, $\mathrm{CH}_{3}$ of phenyl ring), $2.60\left(3 \mathrm{H}\right.$, singlet, $\mathrm{CH}_{3}$ of triazole ring), 7.32-8.29 (10H, multiplet, aromatic protons), $11.49\left(1 \mathrm{H}\right.$, singlet, $-\mathrm{OH}$ proton). ${ }^{13} \mathrm{C} \mathrm{NMR}\left(100 \mathrm{MHz}, \mathrm{CDCl}_{3}, \delta\right): 10.49\left(\mathrm{CH}_{3}\right)$, $21.59\left(\mathrm{CH}_{3}\right), 111.18(\mathrm{CH}), 113.63(\mathrm{CH}), 117.72(\mathrm{CH}), 123.80(\mathrm{CH}), 125.09(\mathrm{CH}), 125.32(\mathrm{CH})$, 130.23(CH), 130.88(CH), 131.17(C), 131.64(C), 132.05(C), 133.39(C), 135.73(C), 140.22(C), 140.35(C), 142.93(C), 145.17(C), 150.78(C), 162.70(CO of coumarin).

7-Hydroxy-9-[1'-(p-methoxyphenyl)-5'-methyl-1H-1,2,3-triazole-4'-yl]benzo[c]coumarin (3c): White solid; yield $=74 \%$; mp $245-247^{\circ} \mathrm{C}$; Anal. Calcd. For $\mathrm{C}_{23} \mathrm{H}_{17} \mathrm{~N}_{3} \mathrm{O}_{4}$ : C, 69.17; H, 4.29; N, $10.52 \%$. Found: C, 69.22; H, 4.35; N, 10.56\%. IR (KBr, $\left.v_{\max }, \mathrm{cm}^{-1}\right) ; 1674(\mathrm{C}=\mathrm{O}$ stretching of $\delta-$ lactone of coumarin), 1620 (aromatic $\mathrm{C}=\mathrm{C}$ stretching), 3063 (aromatic $\mathrm{C}-\mathrm{H}$ stretching), 2932 
(aliphatic C-H stretching), 3418 (O-H stretching). ${ }^{1} \mathrm{H}$ NMR (400MHz, $\left.\mathrm{CDCl}_{3}, \delta\right): 2.59(3 \mathrm{H}$, singlet, $\left.\mathrm{CH}_{3}\right), 3.93\left(3 \mathrm{H}\right.$, singlet, $\left.\mathrm{OCH}_{3}\right), 7.09-8.31(10 \mathrm{H}$, multiplet, aromatic protons $), 11.50(1 \mathrm{H}$, singlet, -OH proton). ${ }^{13} \mathrm{C}$ NMR $\left(100 \mathrm{MHz}, \mathrm{CDCl}_{3}, \delta\right): 10.65\left(\mathrm{CH}_{3}\right), 55.71\left(\mathrm{OCH}_{3}\right), 111.09(\mathrm{CH})$, $112.32(\mathrm{C}) \quad 113.65(\mathrm{CH}), \quad 114.76(\mathrm{CH}), \quad 117.69(\mathrm{CH}), \quad 118.48(\mathrm{C}), \quad 123.77(\mathrm{CH}), \quad 125.35(\mathrm{CH})$, 126.85(CH), 128.67(C), 130.81(CH), 131.75(C), 134.60(C), 135.86(C), 140.53(C), 142.19(C), 150.88(C), 160.68(C), 162.74(CO of coumarin).

7-Hydroxy-9-(1'-(p-chlorophenyl)-5'-methyl-1H-1,2,3-triazole-4'-yl]benzo[c]coumarin

(3d): White solid; yield $=81 \%$; mp 218-220 ${ }^{\circ} \mathrm{C}$; Anal. Calcd. For $\mathrm{C}_{22} \mathrm{H}_{14} \mathrm{ClN}_{3} \mathrm{O}_{3}: \mathrm{C}, 65.43 ; \mathrm{H}, 3.49 ; \mathrm{N}$, $10.41 \%$. Found: C, 65.47; H, 3.45; N, 10.46\%. IR (KBr, $\left.v_{\max }, \mathrm{cm}^{-1}\right) ; 1713(\mathrm{C}=\mathrm{O}$ stretching of $\delta-$ lactone of coumarin), 1636 (aromatic $\mathrm{C}=\mathrm{C}$ stretching), 3094 (aromatic $\mathrm{C}-\mathrm{H}$ stretching), 2962 (aliphatic C-H stretching), 3425 (O-H stretching). ${ }^{1} \mathrm{H} \mathrm{NMR}\left(400 \mathrm{MHz}, \mathrm{CDCl}_{3}, \delta\right): 2.62(3 \mathrm{H}$, singlet, $\left.\mathrm{CH}_{3}\right), 7.28-8.27(10 \mathrm{H}$, multiplet, aromatic protons $), 11.49(1 \mathrm{H}$, singlet, -OH proton $) .{ }^{13} \mathrm{C}$ NMR $\left(100 \mathrm{MHz}, \mathrm{CDCl}_{3}, \delta\right): 10.70\left(\mathrm{CH}_{3}\right), 105.19(\mathrm{C}), 111.13(\mathrm{CH}), 113.69(\mathrm{CH}), 117.79(\mathrm{CH})$, $118.28(\mathrm{C}), \quad 123.73(\mathrm{CH}), \quad 125.30(\mathrm{CH}), \quad 126.65(\mathrm{CH}), \quad 130.00(\mathrm{CH}), \quad 130.92(\mathrm{CH}), \quad 131.51(\mathrm{C})$, 134.37(C), 135.79(C), 136.10(C), 139.99(C), 143.27(C), 150.78(C), 162.66(C), 165.29(CO of coumarin).

7-Hydroxy-4-methoxy-9-[5'-methyl-1'-phenyl-1 H-1,2,3-triazole-4'-yl]benzo[c]coumarin (3e): White solid; yield $=78 \%$; mp $257-259^{\circ} \mathrm{C}$; Anal. Calcd. For $\mathrm{C}_{23} \mathrm{H}_{17} \mathrm{~N}_{3} \mathrm{O}_{4}$ : C, 69.17; $\mathrm{H}, 4.29 ; \mathrm{N}$, $10.52 \%$. Found: C, $69.23 ; \mathrm{H}, 4.33$; N, 10.58\%. IR (KBr, $\left.v_{\max }, \mathrm{cm}^{-1}\right) ; 1682(\mathrm{C}=\mathrm{O}$ stretching of $\delta$ lactone of coumarin), 1628 (aromatic $\mathrm{C}=\mathrm{C}$ stretching), 3070 (aromatic $\mathrm{C}-\mathrm{H}$ stretching), 2978 (aliphatic C-H stretching), 3425 (O-H stretching). ${ }^{1} \mathrm{H}$ NMR $\left(400 \mathrm{MHz}, \mathrm{CDCl}_{3}, \delta\right): 2.61(3 \mathrm{H}$, singlet, $\left.\mathrm{CH}_{3}\right), 4.00\left(3 \mathrm{H}\right.$, singlet, $\left.\mathrm{OCH}_{3}\right), 6.90-8.40(10 \mathrm{H}$, multiplet, aromatic protons $), 11.42(1 \mathrm{H}$, singlet, -OH proton). ${ }^{13} \mathrm{C}$ NMR $\left(100 \mathrm{MHz}, \mathrm{CDCl}_{3}, \delta\right): 10.78\left(\mathrm{CH}_{3}\right), 56.28\left(\mathrm{OCH}_{3}\right), 105.05(\mathrm{C})$, $111.55(\mathrm{CH}), \quad 112.54(\mathrm{CH}), \quad 113.68(\mathrm{CH}), \quad 114.99(\mathrm{CH}), 119.10(\mathrm{C}), 124.70(\mathrm{CH}), 124.93(\mathrm{CH})$, 125.46(C), 129.71(CH), 129.94(CH), 131.63(C), 135.86(C), 135.90(C), 140.11(C), 143.00(C), 148.03(C), 162.56(C), 164.74(CO of coumarin).

7-Hydroxy-4-methoxy-9-[5'-methyl-1'-(p-tolyl)-1H-1,2,3-triazole-4'-yl]benzo[c]coumarin (3f): White solid; yield $=76 \%$; mp $197-199^{\circ} \mathrm{C}$; Anal. Calcd. For $\mathrm{C}_{24} \mathrm{H}_{19} \mathrm{~N}_{3} \mathrm{O}_{4}$ : C, 69.72; $\mathrm{H}, 4.63 ; \mathrm{N}$, $10.16 \%$. Found: C, 69.76; H, 4.68; N, 10.21\%. IR (KBr, $\left.v_{\max }, \mathrm{cm}^{-1}\right) ; 1713(\mathrm{C}=\mathrm{O}$ stretching of $\delta-$ lactone of coumarin), 1636 (aromatic $\mathrm{C}=\mathrm{C}$ stretching), 3032 (aromatic $\mathrm{C}-\mathrm{H}$ stretching), 2939 (aliphatic C-H stretching), 3418 (O-H stretching). ${ }^{1} \mathrm{H}$ NMR $\left(400 \mathrm{MHz}, \mathrm{CDCl}_{3}, \delta\right): 2.60(3 \mathrm{H}$, singlet, $\mathrm{CH}_{3}$ of phenyl ring), $2.61\left(3 \mathrm{H}\right.$, singlet, $\mathrm{CH}_{3}$ of triazole ring), $4.01\left(3 \mathrm{H}\right.$, singlet, $\left.\mathrm{OCH}_{3}\right)$, 7.19-8.26 (9H, multiplet, aromatic protons), $11.51(1 \mathrm{H}$, singlet, $-\mathrm{OH}$ proton $) .{ }^{13} \mathrm{C} \mathrm{NMR}(100 \mathrm{MHz}$, $\left.\mathrm{CDCl}_{3}, \delta\right): 10.33\left(\mathrm{CH}_{3}\right), \quad 21.51\left(\mathrm{CH}_{3}\right), \quad 56.22\left(\mathrm{OCH}_{3}\right), \quad 102.40(\mathrm{C}), 116.08(\mathrm{CH}), 119.74(\mathrm{CH})$, $120.16(\mathrm{CH}), \quad 120.31(\mathrm{C}), \quad 122.64(\mathrm{CH}), \quad 125.16(\mathrm{CH}), \quad 127.17(\mathrm{C}), \quad 130.09(\mathrm{CH}), \quad 130.25(\mathrm{CH})$, 132.21(C), 133.75(C), 139.48(C), 139.78(C), 142.90(C), 144.57(C), 146.93(C), 152.71(C), 162.61(CO of coumarin).

7-Hydroxy-4-methoxy-9-[1'-(p-methoxyphenyl)-5'-methyl-1H-1,2,3-triazole-4'-yl]benzo[c]coumarin (3g): White solid; yield $=85 \%$; mp 248-250 ${ }^{\circ} \mathrm{C}$; Anal. Calcd. For $\mathrm{C}_{24} \mathrm{H}_{19} \mathrm{~N}_{3} \mathrm{O}_{5}: \mathrm{C}, 67.13 ; \mathrm{H}$, 4.46; N, 9.79\%. Found: C, 67.08; H, 4.42; N, 9.75\%. IR (KBr, $\left.v_{\max }, \mathrm{cm}^{-1}\right) ; 1682(\mathrm{C}=\mathrm{O}$ stretching of $\delta$-lactone of coumarin), 1597 (aromatic $\mathrm{C}=\mathrm{C}$ stretching), 3032 (aromatic $\mathrm{C}-\mathrm{H}$ stretching), 2939 (aliphatic C-H stretching), 3448 (O-H stretching). ${ }^{1} \mathrm{H}$ NMR $\left(400 \mathrm{MHz}, \mathrm{CDCl}_{3}, \delta\right): 2.69(3 \mathrm{H}$, singlet, $\left.\mathrm{CH}_{3}\right), 3.40$ and $4.05\left(6 \mathrm{H}\right.$, two singlets, $\mathrm{OCH}_{3}$ of phenyl ring and $\mathrm{OCH}_{3}$ of phenyl ring of coumarin), 7.20-8.07 (9H, multiplet, aromatic protons), 10.84 (1 H, singlet, -OH proton). ${ }^{13} \mathrm{C}$ NMR $\left(100 \mathrm{MHz}, \mathrm{CDCl}_{3}, \delta\right): 10.17\left(\mathrm{CH}_{3}\right), 55.95\left(\mathrm{OCH}_{3}\right), 56.52\left(\mathrm{OCH}_{3}\right), 110.00(\mathrm{C}), 112.61(\mathrm{CH})$, 112.83(C), 114.05(CH), 114.82(CH), 114.98(CH), 115.60(CH), 115.66(C), 117.54(C), 118.09(C), 118.50(C), 126.11(CH), 126.70(CH), 137.55(C), 140.91(C) 147.75(C), 151.60(C), 161.01(C), 162.47 (CO of coumarin). 
7-Hydroxy-4-methoxy-9-(1'-(p-chloroyphenyl)-5'-methyl-1 H-1,2,3-triazole-4'-yl]benzo[c]coumarin (3h): White solid; yield $=79 \%$; mp 267-269 ${ }^{\circ} \mathrm{C}$; Anal. Calcd. For $\mathrm{C}_{23} \mathrm{H}_{16} \mathrm{ClN}_{3} \mathrm{O}_{4}$ : C, 63.67; $\mathrm{H}$, 3.72; N, 9.69\%. Found: C, 63.71; H, 3.76; N, 9.75\%. IR $\left(\mathrm{KBr}, v_{\max }, \mathrm{cm}^{-1}\right) ; 1713(\mathrm{C}=\mathrm{O}$ stretching of $\delta$-lactone of coumarin), 1597 (aromatic $\mathrm{C}=\mathrm{C}$ stretching), 3032 (aromatic $\mathrm{C}-\mathrm{H}$ stretching), 2939 (aliphatic C-H stretching), 3448 (O-H stretching). ${ }^{1} \mathrm{H}$ NMR $\left(400 \mathrm{MHz}, \mathrm{CDCl}_{3}, \delta\right): 2.61(3 \mathrm{H}$, singlet, $\left.\mathrm{CH}_{3}\right), 4.02\left(3 \mathrm{H}\right.$, singlet, $\left.\mathrm{OCH}_{3}\right), 7.09-8.25(9 \mathrm{H}$, multiplet, aromatic protons $), 11.52(1 \mathrm{H}$, singlet, $-\mathrm{OH}$ proton). ${ }^{13} \mathrm{C}$ NMR $\left(100 \mathrm{MHz}, \mathrm{CDCl}_{3}, \delta\right): 10.90\left(\mathrm{CH}_{3}\right), 56.50\left(\mathrm{OCH}_{3}\right), 105.20(\mathrm{C})$, $111.66(\mathrm{CH}), \quad 112.50(\mathrm{CH}), \quad 113.70(\mathrm{CH}), \quad 114.95(\mathrm{CH}), \quad 119.12(\mathrm{C}), \quad 125.01(\mathrm{CH}), \quad 126.68(\mathrm{CH})$, 128.29(C), 130.00(CH), 131.49(C), 134.37(C), 135.82(C), 135.96(C), 136.10(C), 140.00(C), 148.12(C), 148.98(C), 162.63(CO of coumarin).

2-Bromo-7-hydroxy-9-[5'-methyl-1'-phenyl-1H-1,2,3-triazole-4'-yl]benzo[c]coumarin

(3i): White solid; yield $=75 \%$; mp 244-246 ${ }^{\circ} \mathrm{C}$; Anal. Calcd. For $\mathrm{C}_{22} \mathrm{H}_{14} \mathrm{BrN}_{3} \mathrm{O}_{3}: \mathrm{C}, 58.95 ; \mathrm{H}, 3.15 ; \mathrm{N}$, 9.37\%. Found: C, $58.91 ; \mathrm{H}, 3.11 ; \mathrm{N}, 9.32 \%$. IR $\left(\mathrm{KBr}, v_{\max }, \mathrm{cm}^{-1}\right) ; 1713(\mathrm{C}=\mathrm{O}$ stretching of $\delta-$ lactone of coumarin), 1628 (aromatic $\mathrm{C}=\mathrm{C}$ stretching), 3078 (aromatic $\mathrm{C}-\mathrm{H}$ stretching), 3009 (aliphatic C-H stretching), 3448 (O-H stretching). ${ }^{1} \mathrm{H}$ NMR $\left(400 \mathrm{MHz}, \mathrm{CDCl}_{3}, \delta\right): 2.62(3 \mathrm{H}$, singlet, $\left.\mathrm{CH}_{3}\right), 7.25-8.28(10 \mathrm{H}$, multiplet, aromatic protons $), 11.35(1 \mathrm{H}$, singlet, $-\mathrm{OH}$ proton $) .{ }^{13} \mathrm{C}$ NMR $\left(100 \mathrm{MHz}, \mathrm{CDCl}_{3}, \delta\right): 10.76\left(\mathrm{CH}_{3}\right), 104.80(\mathrm{C}), 111.19(\mathrm{CH}), 114.24(\mathrm{CH}), 118.35(\mathrm{C})$, 119.42(CH), $120.05(\mathrm{C}), \quad 125.47(\mathrm{CH}), \quad 126.53(\mathrm{CH}), \quad 129.73(\mathrm{CH}), \quad 130.01(\mathrm{CH}), \quad 131.79(\mathrm{C})$, 133.65(CH), 134.32(C), 135.82(C), 140.43(C), 142.66(C), 149.57(C), 162.65(C), 164.70(CO of coumarin).

2-Bromo-7-hydroxy-9-[5'-methyl-1'-(p-tolyl)-1H-1,2,3-triazole-4'-yl]benzo[c]coumarin (3j): White solid; yield $=71 \%$; mp 252-254 ${ }^{\circ} \mathrm{C}$; Anal. Calcd. For $\mathrm{C}_{23} \mathrm{H}_{16} \mathrm{BrN}_{3} \mathrm{O}_{3}$ : C, 59.76; H, 3.49; N, 9.09\%. Found: $\mathrm{C}, 59.80 ; \mathrm{H}, 3.53 ; \mathrm{N}, 9.13 \%$. IR $\left(\mathrm{KBr}, v_{\max }, \mathrm{cm}^{-1}\right) ; 1682(\mathrm{C}=\mathrm{O}$ stretching of $\delta-$ lactone of coumarin), 1628 (aromatic $\mathrm{C}=\mathrm{C}$ stretching), 3078 (aromatic $\mathrm{C}-\mathrm{H}$ stretching), 2901 (aliphatic C-H stretching), 3441 (O-H stretching). ${ }^{1} \mathrm{H}$ NMR $\left(400 \mathrm{MHz}, \mathrm{CDCl}_{3}, \delta\right): 2.50(3 \mathrm{H}$, singlet, $\mathrm{CH}_{3}$ of phenyl ring), $2.60\left(3 \mathrm{H}\right.$, singlet, $\mathrm{CH}_{3}$ of triazole ring), 7.27-8.31 (9H, multiplet, aromatic protons), 11.38 (1H, singlet, $-\mathrm{OH}$ proton). ${ }^{13} \mathrm{C} \mathrm{NMR}\left(100 \mathrm{MHz}, \mathrm{CDCl}_{3}, \delta\right): 10.75\left(\mathrm{CH}_{3}\right)$, $21.33\left(\mathrm{CH}_{3}\right), \quad 104.81(\mathrm{C}), \quad 111.21(\mathrm{CH}), \quad 114.24(\mathrm{CH}), \quad 118.36(\mathrm{C}), \quad 119.44(\mathrm{CH}), \quad 120.09(\mathrm{C})$, 125.28(CH), 126.57(CH), 130.28(CH), 131.83(C), 133.30(C), 133.66(CH), 134.35(C), 140.32(C), 140.54(C), 142.56(C), 149.59(C), 162.68(C), 164.77(CO of coumarin).

2-Bromo-7-hydroxy-9-[1'-(p-methoxyphenyl)-5'-methyl-1H-1,2,3-triazole-4'-yl]benzo[c]coumarin (3k): White solid; yield $=71 \%$; mp $234-236^{\circ} \mathrm{C}$; Anal. Calcd. For $\mathrm{C}_{23} \mathrm{H}_{16} \mathrm{BrN}_{3} \mathrm{O}_{4}$ : C, 57.76; $\mathrm{H}$, 3.37; N, 8.79\%. Found: C, 57.81; H, 3.42; N, 8.74\%. IR $\left(\mathrm{KBr}, v_{\max }, \mathrm{cm}^{-1}\right) ; 1713(\mathrm{C}=\mathrm{O}$ stretching of $\delta$-lactone of coumarin), 1628 (aromatic $\mathrm{C}=\mathrm{C}$ stretching), 3070 (aromatic $\mathrm{C}$-H stretching), 2970 (aliphatic C-H stretching), 3448 (O-H stretching). ${ }^{1} \mathrm{H}$ NMR $\left(400 \mathrm{MHz}, \mathrm{CDCl}_{3}, \delta\right): 2.59(3 \mathrm{H}$, singlet, $\left.\mathrm{CH}_{3}\right) 3.91\left(3 \mathrm{H}\right.$, singlet, $\left.\mathrm{OCH}_{3}\right), 7.10-8.33(9 \mathrm{H}$, multiplet, aromatic protons $), 11.39(1 \mathrm{H}$, singlet, -OH proton). ${ }^{13} \mathrm{C}$ NMR $\left(100 \mathrm{MHz}, \mathrm{CDCl}_{3}, \delta\right): 10.72\left(\mathrm{CH}_{3}\right), 55.73\left(\mathrm{OCH}_{3}\right), 104.75(\mathrm{C})$, 111.29(CH), 114.24(CH), 114.98(CH), 118.41(C), 119.48(CH), 120.05(C), 126.52(CH), 127.01(CH), 128.81(C), 132.08(C), 133.80(CH), 134.45(C), 140.76(C), 142.47(C), 149.59(C), 153.11(C), 160.64(C), 162.69(CO of coumarin).

2-Bromo-7-hydroxy-9-[1'-(p-chlorophenyl)-5'-methyl-1H-1,2,3-triazole-4'-yl]benzo[c]coumarin (3I): White solid; yield $=76 \%$; mp $291-293^{\circ} \mathrm{C}$; Anal. Calcd. For $\mathrm{C}_{23} \mathrm{H}_{14} \mathrm{BrClN}_{3} \mathrm{O}_{3}$ : C, 54.74; $\mathrm{H}$, $2.71 ; \mathrm{N}, 8.70 \%$. Found: C, $54.70 ; \mathrm{H}, 2.67 ; \mathrm{N}, 8.65 \%$. IR $\left(\mathrm{KBr}, v_{\max }, \mathrm{cm}^{-1}\right) ; 1705(\mathrm{C}=\mathrm{O}$ stretching of $\delta$-lactone of coumarin), 1628 (aromatic $\mathrm{C}=\mathrm{C}$ stretching), 3078 (aromatic $\mathrm{C}$-H stretching), 3009 (aliphatic C-H stretching), 3448 (O-H stretching). ${ }^{1} \mathrm{H}$ NMR $\left(400 \mathrm{MHz}, \mathrm{CDCl}_{3}, \delta\right): 2.68(3 \mathrm{H}$, singlet, $\left.\mathrm{CH}_{3}\right), 7.34-8.26\left(9 \mathrm{H}\right.$, multiplet, aromatic protons), $10.87\left(1 \mathrm{H}\right.$, singlet, $-\mathrm{OH}$ proton). ${ }^{13} \mathrm{C}$ NMR $\left(100 \mathrm{MHz}, \mathrm{CDCl}_{3}, \delta\right): 10.14\left(\mathrm{CH}_{3}\right), 109.97(\mathrm{C}), 112.19(\mathrm{CH}), 112.80(\mathrm{C}), 115.56(\mathrm{CH})$, 115.63(C), 118.46(C), 119.07(C), 119.37(C), 119.59(CH), 126.43(CH), 126.58(CH), 130.72(CH), 132.25(C), 134.67(C), 134.89(CH), 135.89(C), 138.61(C), 149.27(C), 165.58(CO of coumarin). 


\section{Results and discussion}

\subsection{Chemistry}

With a view to synthesizing some new $[1,2,3]$ triazolyl benzo[c]coumarins adopting new synthetic route the present work was carried out. In the present work, various 7-hydroxy-9-(5methyl-1-aryl-1H-1,2,3-triazol-4-yl) benzo[c]coumarins (3a-l) have been synthesized by reacting appropriate 3-coumarinoyl methyl pyridinium bromide salt (1a-c) with various 1-(5- methyl-1-aryl$1 H$-1,2,3-triazol-4-yl)ethanones (2a-d) in the presence of sodium acetate in refluxing acetic acid. The required triazole derivatives (2a-d) were prepared by reacting appropriate aryl azide with acetyl acetone in the presence of potassium carbonate (Scheme-1). The structures of all the synthesized compounds (3a-l) were established by IR, ${ }^{1} \mathrm{H}-\mathrm{NMR},{ }^{13} \mathrm{C}-\mathrm{NMR}$ and selected mass spectral data are shown in experimental section 2.2.
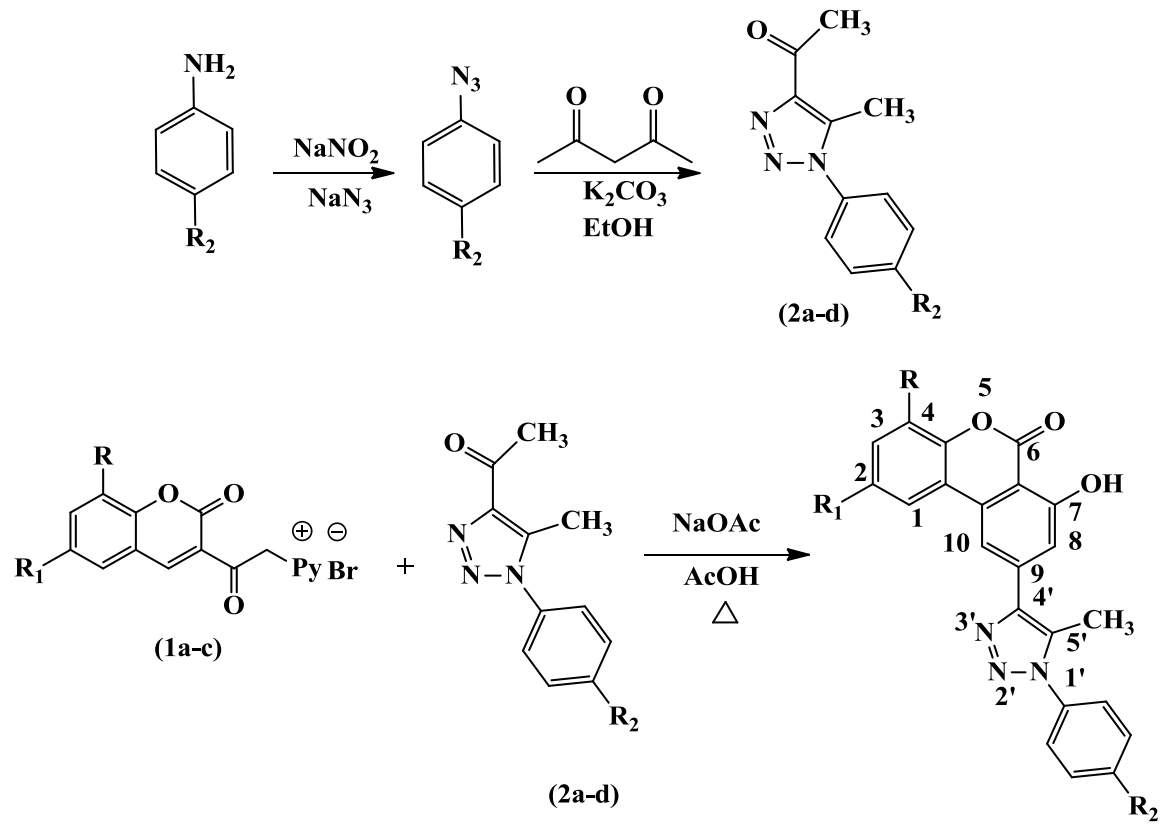

(2a-d)

(3a-l)

\begin{tabular}{|c|c|c|c|c|c|c|c|}
\hline Compound & $\mathbf{R}$ & $\mathbf{R}_{\mathbf{1}}$ & $\mathbf{R}_{\mathbf{2}}$ & Compound & $\mathbf{R}$ & $\mathbf{R}_{\mathbf{1}}$ & $\mathbf{R}_{\mathbf{2}}$ \\
\hline $\mathbf{3 a}$ & $\mathrm{H}$ & $\mathrm{H}$ & $\mathrm{H}$ & $\mathbf{3 g}$ & $\mathrm{OCH}_{3}$ & $\mathrm{H}$ & $\mathrm{OCH}_{3}$ \\
\hline $\mathbf{3 b}$ & $\mathrm{H}$ & $\mathrm{H}$ & $\mathrm{CH}_{3}$ & $\mathbf{3 h}$ & $\mathrm{OCH}_{3}$ & $\mathrm{H}$ & $\mathrm{Cl}$ \\
\hline $\mathbf{3 c}$ & $\mathrm{H}$ & $\mathrm{H}$ & $\mathrm{OCH}_{3}$ & $\mathbf{3 i}$ & $\mathrm{H}$ & $\mathrm{Br}$ & $\mathrm{H}$ \\
\hline $\mathbf{3 d}$ & $\mathrm{H}$ & $\mathrm{H}$ & $\mathrm{Cl}$ & $\mathbf{3 j}$ & $\mathrm{H}$ & $\mathrm{Br}$ & $\mathrm{CH}_{3}$ \\
\hline $\mathbf{3 e}$ & $\mathrm{OCH}_{3}$ & $\mathrm{H}$ & $\mathrm{H}$ & $\mathbf{3 k}$ & $\mathrm{H}$ & $\mathrm{Br}$ & $\mathrm{OCH}_{3}$ \\
\hline $\mathbf{3 f}$ & $\mathrm{OCH}_{3}$ & $\mathrm{H}$ & $\mathrm{CH}_{3}$ & $\mathbf{3 l}$ & $\mathrm{H}$ & $\mathrm{Br}$ & $\mathrm{Cl}$ \\
\hline
\end{tabular}

Scheme 1: Synthetic scheme for the compounds (3a-1)

\subsection{Mechanism}

It is reported that reaction of $\alpha, \beta$ - unsaturated ketone with methyl ketone or ketone with active methylene group in the presence of sodium acetate results in a formation of 1,3,5- tri substituted benzene derivatives [42]. This novel reaction is utilized in the preparation of present compounds. Here the benzene ring has been built up between 3 and 4 position of coumarin nucleus by utilizing this reaction. The plausible mechanism for the synthesis of target compounds (3a-I) is shown in Scheme-2. The reaction proceeds by the anion generation from the acetyl group of compound (2a-d). This anion further adds to the 3,4-double bond of coumarin (1a-c) and results in the formation of intermediate (A) having 1,5-dione functionality. The active methylene group in (A) then gets cyclized with carbonyl group of [1,2,3]-triazole moiety resulting in the formation of intermediate (B) which finally gets converted into the product by loss of water molecule and subsequent aromatization. The reaction is smooth and results the triazolyl substituted benzo[c]coumarins (3a-l) in good yield. 


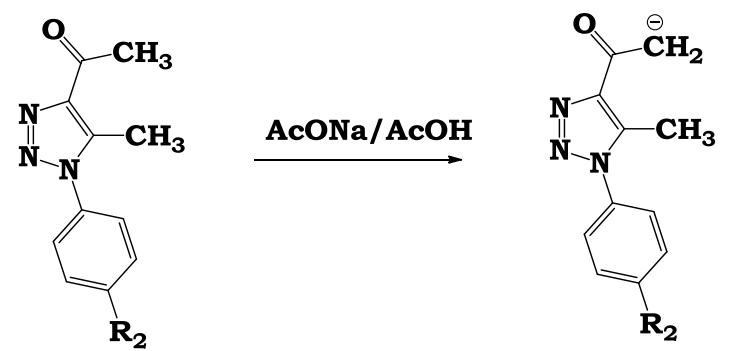

(2a-d)

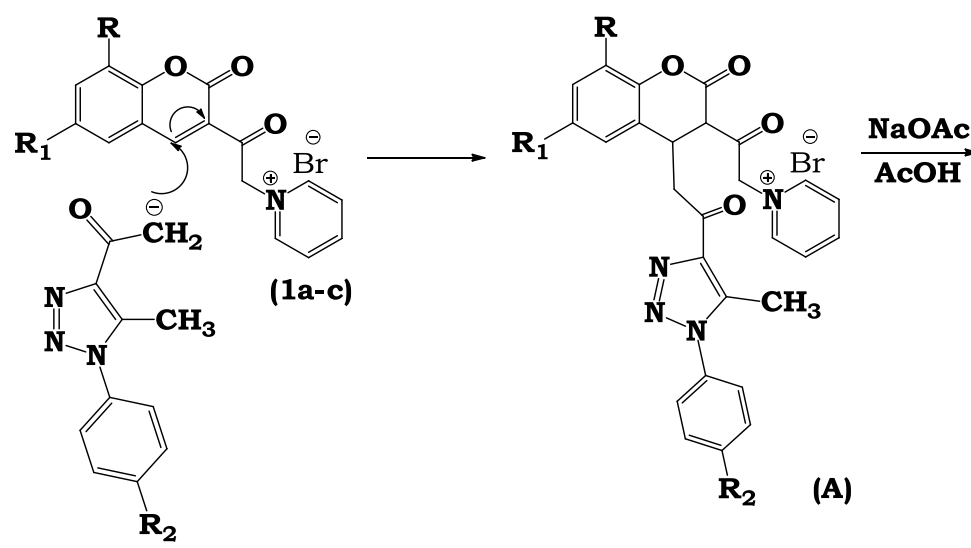

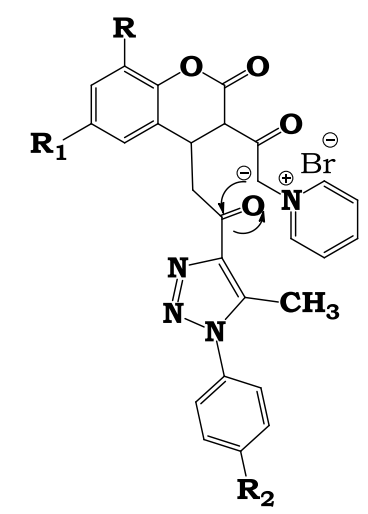

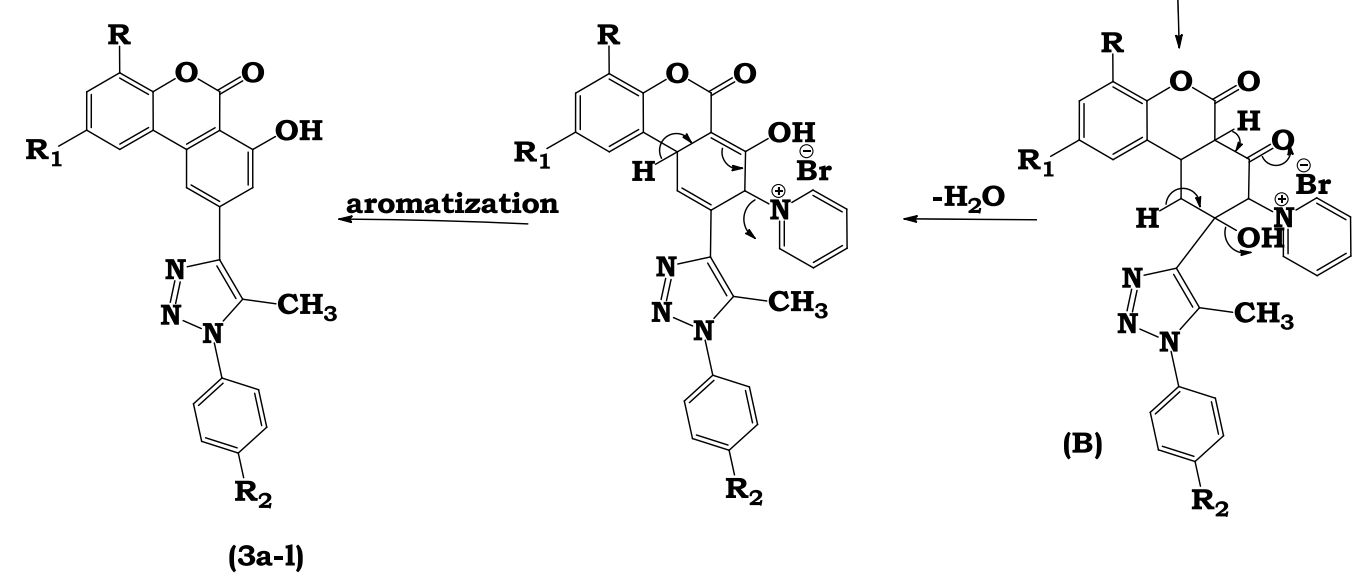

Scheme 2: Plausible mechanism for the synthesis of target compounds (3a-1)

\subsection{Biological results}

\subsubsection{Antimicrobial activity}

The newly synthesized target compounds (3a-l) were evaluated for their in vitro antibacterial activity against two Gram positive bacteria Staphylococcus aureus (MTCC 96) and Bacillus subtilis (MTCC 441) and two Gram negative bacteria Escherichia coli (MTCC 443) and Salmonella typhi (MTCC 98). They were also evaluated for their in vitro antifungal activity against Candida albicans (MTCC 227) and Aspergillus niger (MTCC 282) as fungal strains. Broth dilution method was used for the determination of the antibacterial and antifungal activity as recommended by NCCLS [43]. Ampicillin, Chloramphenicol and Norfloxacin were used as standard antibacterial drugs, whereas Griseofulvin and Nystatin were used as standard antifungal drugs. All MTCC cultures were collected from Institute of Microbial Technology, Chandigarh and tested against above mentioned known drugs. Mueller-Hinton broth was used as the nutrient medium for the test bacteria and Sabouraud Dextrose broth was used for the test fungi. Inoculum size for the test strains was adjusted to $10^{8} \mathrm{CFU}$ (Colony Forming Unit per milliliter) per milliliter by comparing the turbidity. Each synthesized compound was diluted with DMSO so as to have the stock solution of $2000 \mu \mathrm{g} / \mathrm{mL}$ concentration as a stock solution. The results were recorded in the 
form of primary and secondary screening. The synthesized compounds (3a-l) were screened for their antibacterial and antifungal activity at the concentration of 1000,500 and $250 \mu \mathrm{g} / \mathrm{mL}$ for the primary screening. The synthesized compound showing activity against microbes in the primary screening were further screened in a second set of dilution at concentrations of 200, 100, 62.5, 50 and $25 \mu \mathrm{g} / \mathrm{mL}$. The suspention of $10 \mu \mathrm{L}$ from each well were further incubated and growth was noted at $37^{\circ} \mathrm{C}$ after 24 hour for bacteria and 48 hour for fungi. The lowest concentration which showed no visible growth (turbidity) after spot subculture was considered as the minimum inhibitory concentration (MIC) for each compound.

The investigation of the data summarized in (Table-1) reveals that many compounds were found to be active against Gram-positive bacteria while some of the compounds were found to be active against Gram-negative bacterial and fungal species as compared to that of the standard antimicrobial drugs.

\subsubsection{Antimicrobial evaluation}

The compounds (3a-l) were screened for their in vitro antibacterial and antifungal evaluation against various bacterial and fungal pathogens by broth dilution method. Ampicillin, Chloramphenicol, Norfloxacin, Griseofulvin and Nystatin were used as standard drugs. The values of MIC are summarized in Table-1.

Review of the antimicrobial activities of synthesized compounds (3a-l) in (Table-1) indicated that compounds $3 \mathbf{e}(\mathrm{MIC}=100, \mu \mathrm{g} / \mathrm{mL})$ exhibited excellent activity toward Gram-positive bacteria Bacillus subtilis as compared to Ampicillin ( $\mathrm{MIC}=250, \mu \mathrm{g} / \mathrm{mL}$ ) and showed equipotent activity to Norfloxacin ( $\mathrm{MIC}=100, \mu \mathrm{g} / \mathrm{mL}$ ). Against Gram-positive bacteria Bacillus subtilis, compound 3k $(\mathrm{MIC}=150, \mu \mathrm{g} / \mathrm{mL})$ showed activity higher than that of Ampicillin ( $\mathrm{MIC}=250$, $\mu \mathrm{g} / \mathrm{mL})$. Compounds 3b, 3f and $\mathbf{3 j}(\mathrm{MIC}=200, \mu \mathrm{g} / \mathrm{mL})$ displayed better activity than Ampicillin $(\mathrm{MIC}=250, \mu \mathrm{g} / \mathrm{mL})$ toward Gram-positive bacteria Bacillus subtilis. Compounds 3a, 3h and 3i $(\mathrm{MIC}=250, \mu \mathrm{g} / \mathrm{mL})$ showed results equivalent to that of Ampicillin $(\mathrm{MIC}=250, \mu \mathrm{g} / \mathrm{mL})$ toward Gram-positive bacteria Bacillus subtilis. Against Gram-positive bacteria Staphylococcus aureus, compound 3e $(\mathrm{MIC}=62.5, \mu \mathrm{g} / \mathrm{mL})$ showed excellent activity as compared to Ampicillin (MIC=250, $\mu \mathrm{g} / \mathrm{mL})$. Compounds $\mathbf{3 f}$ and $\mathbf{3 k}(\mathrm{MIC}=100, \mu \mathrm{g} / \mathrm{mL})$ were found to be more effective against Grampositive bacteria Staphylococcus aureus than Ampicillin $(\mathrm{MIC}=250, \mu \mathrm{g} / \mathrm{mL})$. Compounds $\mathbf{3 b}, \mathbf{3 g}$ and 3j $(\mathrm{MIC}=200, \mu \mathrm{g} / \mathrm{mL})$ showed good activity against Gram-positive bacteria Staphylococcus aureus as compared to Ampicillin $(\mathrm{MIC}=250, \mu \mathrm{g} / \mathrm{mL})$. Against Gram-positive bacteria Staphylococcus aureus, compound 3a and $3 \mathbf{i}(\mathrm{MIC}=250, \mu \mathrm{g} / \mathrm{mL})$ showed equipotent activity to that of Ampicillin ( $\mathrm{MIC}=250, \mu \mathrm{g} / \mathrm{mL})$.

Moreover, Against Gram-negative bacteria Escherichia coli, compounds $3 \mathbf{i}$ and $\mathbf{3 j}$ $(\mathrm{MIC}=62.5, \mu \mathrm{g} / \mathrm{mL})$ showed excellent activity as compared to Ampicillin $(\mathrm{MIC}=100, \mu \mathrm{g} / \mathrm{mL})$. Where as compounds $\mathbf{3 a}, \mathbf{3 h}$ and $\mathbf{3 k}(\mathrm{MIC}=100, \mu \mathrm{g} / \mathrm{mL})$ showed activity comparable to Ampicillin $(\mathrm{MIC}=100, \mu \mathrm{g} / \mathrm{mL})$ toward Gram-negative bacteria Escherichia coli. Against Gram-negative bacteria Salmonella typhi, compounds $\mathbf{3 c}, \mathbf{3 h}, \mathbf{3 i}, \mathbf{3 j}$ and $\mathbf{3 k}(\mathrm{MIC}=100, \mu \mathrm{g} / \mathrm{mL})$ were found to be equipotent with Ampicillin (MIC=100, $\mu \mathrm{g} / \mathrm{mL}$ ).

Furthermore, against Candida albicans fungal pathogen, however compound $3 f(\mathrm{MIC}=250$, $\mu \mathrm{g} / \mathrm{mL}$ ) showed better inhibition action as compare to the standard drug Griseofulvin ( $\mathrm{MIC}=500$, $\mu \mathrm{g} / \mathrm{mL})$. Where as compounds $\mathbf{3 b}, \mathbf{3 h}$ and $\mathbf{3 i}(\mathrm{MIC}=500, \mu \mathrm{g} / \mathrm{mL})$ showed activity comparable to Griseofulvin ( $\mathrm{MIC}=500, \mu \mathrm{g} / \mathrm{mL}$ ) against fungal pathogen Candida albicans.

Majority of the synthesized compounds were active against Gram-positive bacteria viz. Bacillus subtilis (MTCC 441) and Staphylococcus aureus (MTCC 96), Gram-negative bacteria viz. Escherichia coli (MTCC 443) and Salmonella typhi (MTCC 98). Some of the synthesized compounds were found sufficiently potent to inhibit fungal pathogen viz. Candida albicans (MTCC 227). 
Table 1: In vitro Antimicrobial activity of compounds (3a-1)

\begin{tabular}{|c|c|c|c|c|c|c|}
\hline \multirow{4}{*}{ Compound } & \multicolumn{6}{|c|}{ Minimum Inhibitory Concentration (MIC, $\mu g \mathrm{~mL}^{-1}$ ) } \\
\hline & \multicolumn{2}{|c|}{ Gram +ve bacteria } & \multicolumn{2}{|c|}{ Gram-ve bacteria } & \multicolumn{2}{|c|}{ Fungi } \\
\hline & B.s. & S.a. & E.c. & S.t. & A.n. & C.a. \\
\hline & MTCC441 & МТСС96 & MTCC443 & МТСС98 & MTCC282 & МТСС227 \\
\hline 3a & 250 & 250 & 100 & 125 & 500 & 1000 \\
\hline $3 \mathbf{b}$ & 200 & 200 & 200 & 200 & 1000 & 500 \\
\hline $3 c$ & 500 & 500 & 500 & 100 & 200 & $>1000$ \\
\hline 3d & 500 & 500 & 250 & 200 & 250 & $>1000$ \\
\hline $3 \mathrm{e}$ & 100 & 62.5 & 250 & 250 & 500 & 1000 \\
\hline 3f & 200 & 100 & 200 & 125 & $>1000$ & 250 \\
\hline $3 g$ & 500 & 200 & 200 & 200 & 1000 & $>1000$ \\
\hline $3 \mathbf{h}$ & 250 & 500 & 100 & 100 & 1000 & 500 \\
\hline $3 \mathbf{i}$ & 250 & 250 & 62.5 & 100 & 1000 & 500 \\
\hline $3 \mathbf{j}$ & 200 & 200 & 62.5 & 100 & 250 & $>1000$ \\
\hline $3 k$ & 150 & 100 & 100 & 100 & 1000 & 1000 \\
\hline 31 & 500 & 500 & 200 & 250 & $>1000$ & 1000 \\
\hline Ampicillin & 250 & 250 & 100 & 100 & - & - \\
\hline Chloramphenicol & 50 & 50 & 50 & 50 & - & - \\
\hline Norfloxacin & 100 & 10 & 10 & 10 & - & - \\
\hline Griseofulvin & - & - & - & - & 100 & 500 \\
\hline Nystatin & - & - & - & - & 100 & 100 \\
\hline
\end{tabular}

\section{Conclusions}

A novel and efficient protocol for the synthesis of a series of [1,2,3]-triazolyl substituted benzo $[c]$ coumarin derivatives was described and the synthesized compounds were screened for their in vitro antimicrobial evaluation. The results indicated that all the synthesized compounds shown good antibacterial activity. In particular, compounds $\mathbf{3 e}, \mathbf{3 f}, \mathbf{3 i}, \mathbf{3} \mathbf{j}$, and $\mathbf{3 k}$ exhibited the more potent inhibitory activity against bacterial and fungal pathogens as compared to other compounds and emerged as potential lead compounds for further investigations.

\section{Acknowledgement}

The authors are thankful to the Head, Department of Chemistry, Sardar Patel University for providing research facilities. Financial assistance to NNG and KNK from the UGC, New Delhi, India, is highly acknowledged.

\section{References}

[1] J. Lou et al., Metabolites from Alternaria fungi and their bioactivities, Molecules. 18 (2013) 5891-5935.

[2] Y. Shirataki, S. Toda, Anti-oxidative effects of dibenzo- $\alpha$-pyrones in fruits of Trapa natans on lipid peroxidation, Nat. Med. 55 (2001) 247-250.

[3] D. Liang et al, Lysilactones A-C, three 6H-dibenzo[b,d]pyran-6-one glycosides from Lysimachia clethroides, total synthesis of lysilactone A, Tetrahedron. 69 (2013) 2093-2097.

[4] S. Ghosal, J. Reddy, V. Lai, Shilajit I: Chemical Constituents, J. Pharm. Sci. 65(5) (1976) 772-773.

[5] S.J. Jeong et al, Hyaluronidase inhibitory active $6 \mathrm{H}$-dibenzo[b,d]pyran-6-ones from the feces of Trogopterus xanthipes, Planta Med. 66(1) (2000) 76-77. 
[6] K. Koch et al., Total Synthesis of Alternariol, J. Org. Chem. 70(8) (2005) 3275-3276.

[7] C. Tamm, Homoisoflavones, a new series of natural products, Arzneim.-Forsch. 22(10) (1972) 1776-1784.

[8] R.W. Pero, D. Harvan, M.C. Blois, Isolation of the toxin, altenuisol, from the fungus, alternariatenuis auct, Tetrahedron Lett. 14(12) (1973) 945-948.

[9] S. Madan, C.H. Cheng, Nickel-Catalyzed Synthesis of Benzocoumarins: Application to the Total Synthesis of Arnottin I, J. Org. Chem. 71(21) (2006) 8312-8315.

[10] A.D. Patten, N.H. Nguyen, S.J. Danishefsky, Concise Total Synthesis of Defucogilvocarcin V, J. Org. Chem. 53(5) (1988) 1003-1007.

[11] J.P. Edwards et al., 5-Aryl-1,2- dihydro-5H-chromeno[3,4-f]quinolines as potent, orally active, nonsteroidal progesterone receptor agonists: The effect of D-ring substituents, J. Med. Chem. 41 (1998) 303-310.

[12] L.G. Hamann et al, Synthesis and biological activity of a novel series of nonsteroidal, peripherally selective androgen receptor antagonists derived from 1,2-dihydropyridono[5,6g]quinolones, J. Med. Chem. 41 (4) (1998) 623-639.

[13] M.J. Coghlan et al, Synthesis and characterization of non-steroidal ligands for the glucocorticoid receptor: Selective quinoline derivatives with prednisolone-equivalent functional activity, J. Med. Chem. 44(18) (2001) 2879-2885.

[14] J.M. Schmidt et al, Synthesis and evaluation of a novel nonsteroidal-specific endothelial cell proliferation inhibitor, J. Med. Chem. 46 (2003) 1289-1292.

[15] K.V. Sashidhara et al, Novel coumarin derivatives as potential antidyslipidemic agents, Biorg. Med. Chem. Lett. 20 (2010) 4248-4251.

[16] M. Morimoto et al., Gilvocarcins, new antitumor antibiotics 3. Antitumor activity, J. Antibiot. 34 (1981) 701-707.

[17] F. Tomita, K. Takahashi, T. Tamaoki, Gilvocarcins, new antitumor antibiotics 4. Mode of action, J. Antibiot. 35(8) (1982) 1038-1041.

[18] H. Nakashima et al, Inhibition of human immunodeficiency viral replication by tannins and related compounds, Anti-Viral Res. 18(1) (1992) 91-103.

[19] T. Okuda, K. Mori, H. Hayatsu, Inhibitory Effect of Tannins on Direct-acting Mutagens, Chem. Pharm. Bull. 32 (1984) 3755-3758.

[20] Z. Qiu et al, In vitro antioxidant and antiproliferative effects of ellagic acid and its colonic metabolite, urolithins, on human bladder cancer T24 cells, Food Chem. Toxicol. 59 (2013) 428-437.

[21] M. Sakurai et al., TMC-264, a novel anti-allergic heptaketide produced by the fungus phoma sp. TC 1674, Org. Letters. 5(7) (2003) 1083-1085.

[22] A.H. Aly et al., Cytotoxic metabolites from the fungal endophyte Alternaria sp. and their subsequent detection in its host plant Polygonum senegalense, J. Nat. Prod. 71(6) (2008) 972980.

[23] E. Hormazabal et al., Metabolites from Microsphaeropsis olivacea, an Endophytic Fungus of Pilgerodendron uviferum, Zeitschrift für Naturforschung C. 60(1-2) (2005) 11-21.

[24] Y.C. Song et al., Characterization of Graphislactone A as the Antioxidant and Free RadicalScavenging Substance from the Culture of Cephalosporium sp. IFB-E001, an Endophytic Fungus in Trachelospermum jasminoides, Biol. Pharm. Bull. 28(3) (2005) 506-509. 
[25] P. Langer, N.N.R. Saleh, B. Appel, Domino Reactions of 1,3-Bis-Silyl Enol Ethers with Benzopyrylium Triflates Efficient Synthesis of Fluorescent 6H-Benzo[c]chromen-6-ones, Dibenzo[c,d]chromen-6-ones, and 2,3-Dihydro-1H-4,6-dioxachrysen-5-ones, Chem. Eur. J. 12 (2006) 1221-1236.

[26] B.I. Alo et al., Sequential directed ortho metalation-boronic acid cross-coupling reactions. A general regiospecific route to oxygenated dibenzo[b,d]pyran-6-ones Related to Ellagic Acid, J. Org. Chem. 56(12) (1991) 3763-3768.

[27] M.S. Tremblay, D. Sames, A New Fluorogenic Transformation: Development of an Optical Probe for Coenzyme Q, Org. Lett. 7(12) (2005) 2417-2420.

[28] R. Alvarez et al., 1,2,3-Triazole-[2,5-Bis-O-(tert-butyldimethylsilyl)-.beta.-D-ribofuranosyl]3'-spiro-5"-(4"'-amino-1",2"-oxathiole2",2"'-dioxide)(TSAO)Analogues: Synthesis and AntiHIV-1 Activity, J Med Chem. 37(24) (1994) 4185-4194.

[29] M.J. Genin et al., Substituent Effects on the Antibacterial Activity of Nitrogen-CarbonLinked (Azolylphenyl)oxazolidinones with Expanded Activity Against the Fastidious GramNegative Organisms Haemophilus influenzae and Moraxella catarrhalis, J. Med. Chem. 43 (2000) 953-970.

[30] D.R. Buckle et al., Studies on 1,2,3-Triazoles. 13. (Piperazinylalkoxy)[1]benzopyrano[2,3-d]1,2,3-triazol-9 $(1 \mathrm{H})$-ones with Combined H1-Antihistamine and Mast Cell Stabilizing Properties, J Med Chem. 29(11) (1986) 2262-2267.

[31] D.J. Hupe et al., The Inhibition of Receptor-mediated and Voltage-dependent Calcium Entry by the Anti-proliferative L-651,582, J Biol Chem. 266(16) (1991) 10136-10142.

[32] O. Makabe, H. Suzuki, S. Umezawa, Synthesis of D-arabinofuranosyl and 2'-deoxy-Dribofuranosyl 1,2,3-Triazolecarboxamides, Bull. Chem. Soc. Jpn. 50 (1977) 2689-2693.

[33] S. Syed et al, Synthesis of novel 2-mercapto benzothiazole and 1,2,3-triazole based bisheterocycles: Their anti-inflammatory and anti-nociceptive activities, Eur. J. Med. Chem. 49 (2012) 324-333.

[34] I.S. Bennett et al., 6-(substituted methylene)penems, potent broad spectrum inhibitors of bacterial beta-lactamase. V. Chiral1, 2,3-triazolyl derivatives, J. Antibiot. 44(9) (1991) 969978.

[35] E.K. Moltzen et al., Bioisosteres of Arecoline: 1,2,3,6-Tetrahydro-5-pyridyl-Substituted and 3-Piperidyl-Substituted Derivatives of Tetrazoles and 1,2,3-Triazoles. Synthesis and Muscarinic Activity, J Med Chem. 37(24) (1994) 4085-4099.

[36] Z. Bascal et al., Novel Azole Derivatives Are Antagonists At The Inhibitory Gaba Receptor On The Somatic Muscle-Cells Of The Parasitic Nematode Ascaris- Suum, Parasitology. 112 (1996) 253-259.

[37] H. Wamhoff et al., 1,2,3-triazoles, Comprehensive Heterocyclic Chemistry, Elsevier Science, Oxford. 4 (1996) 1-126.

[38] C.F. Koelsch, Bromination of 3-Acetocoumarin, J. Am. Chem. Soc. 72(7) (1950) 2993-2995.

[39] T.V.P. Rao, V.R. Rao, A novel synthesis of thiazolyl, imidazolyl 2H-benzopyran-2-ones, Ind. J. Chem. 25 (1986) 413-415.

[40] Apoorva Patel, Synthesis, characterization and antimicrobial activity study of some heterocyclic substituted and heterocyclic fused coumarin derivatives, Ph.D. Thesis, Sardar Patel University, Vallabh Vidyanagar, 2011. 
[41] V.R. Kamalraj, S. Senthil, P. Kannan, One-pot synthesis and the fluorescent behaviour of 4acetyl-5-methyl-1,2,3-triazole regioisomers, Journal of Molecular Structure. 892 (2008) 210215.

[42] Karl Eichinger et al., Neue Synthesen alkylaryl-und diaryl-disubstituierter Phenole und Salicylsäure-ethylester, Synthesis. 12 (1987) 1061-1064.

[43] National Committee for Clinical Laboratory Standards (NCCLS), 940, West Valley Road, Suite 1400, Wayne, Pennsylvania 19087-1898, USA. Performance Standards for Antimicrobial Susceptibility Testing; Twelfth Informational Supplement (ISBN 1-56238-4546), M100-S12 (M7), 2002. 